\title{
Physiological Regulatory Networks: Ecological Roles and Evolutionary Constraints
}

\author{
Alan A. Cohen ${ }^{1}$ \\ Groupe de recherche PRIMUS \\ Department of Family Medicine \\ University of Sherbrooke \\ CHUS-Fleurimont, $12^{\text {ème }}$ Ave $\mathrm{N}$, \\ Sherbrooke, QC J1H 5N4, Canada \\ Lynn B. Martin \\ Department of Integrative Biology \\ University of South Florida \\ 4202 East Fowler Avenue \\ Tampa FL 33620-5200 USA \\ John C. Wingfield \\ Department of Neurobiology, Physiology and Behavior \\ University of California, Davis \\ One Shields Avenue \\ Davis, CA 95616 USA \\ Scott R. McWilliams \\ University of Rhode Island \\ Dept. of Natural Resources Science \\ Coastal Institute in Kingston, \#116 \\ 1 Greenhouse Road \\ Kingston, RI 02881 USA \\ Jennifer A. Dunne \\ Santa Fe Institute \\ 1399 Hyde Park Road \\ Santa Fe, NM 87501 USA \\ Pacific Ecoinformatics and Computational Ecology Lab \\ 1604 McGee Ave. \\ Berkeley, CA 94703 USA
}

${ }^{1}$ Corresponding author: Cohen, A. A. (Alan.Cohen@USherbrooke.ca) 


\begin{abstract}
Ecological and evolutionary physiology has traditionally focused on aspects of physiology one at a time. Here, we discuss the implications of considering physiological regulatory networks (PRNs) as integrated wholes, a perspective that reveals novel roles for physiology in organismal ecology and evolution. For example, evolutionary response to changes in resource abundance might be constrained by the role of dietary micronutrients in immune response regulation, given a particular pathogen environment. Because many physiological components impact more than one process, organismal homeostasis is maintained, individual fitness is determined, and evolutionary change is constrained (or facilitated) by interactions within PRNs. We discuss how PRN structure and its system-level properties could determine both individual performance and patterns of physiological evolution.
\end{abstract}

\title{
Glossary
}

Alternative physiological structures - Different PRN structures that would produce equivalent functional and fitness outcomes for a given species in a given environment. Which structure evolves might be largely random, but might have consequences for long-term evolution of PRN structure.

Cytokines - Small cell-signalling proteins involved in intercellular communication. Regulatory cascades of cytokines are particularly critical in the immune system.

Dysregulation - A breakdown over time in the ability of an individual's PRN to maintain homeostasis; possibly in response to chronic stress or infection, and possibly a root cause of aging. 
Homeostasis - A state of healthy physiological equilibrium, including appropriate anticipation of and responses to changing conditions.

Integrator - A PRN molecule that has a particularly crucial role in synthesizing information (internal or external) and thereby determining multiple aspects of PRN functioning (e.g., a 'hub' or 'keystone' PRN molecule)

Physiological Regulatory Network (PRN) - The network of molecules and their regulatory relationships that maintain and adjust homeostasis and facilitate performance at the wholeorganism level.

PRN Molecule - PRN molecules are the subset of molecules that maintain homeostasis at the organism level via their regulatory relationships with other molecules. In most cases, these molecules are the nodes (vertices) in a PRN, and regulatory relationships between them are the links (edges). In some cases, several related molecules (e.g., circulating steroid, receptors, binding globulins) may be grouped together as a single node for measurement purposes, or gene activation may be a node in some contexts. Flexibility of node definition is common in other biological networks-for example, nodes in ecological networks can be species, groups of species, life stages, or other categories (e.g., detritus).

PRN state - The concentrations of all PRN molecules at a given moment for a given individual in a particular context (e.g. age, time of year, life history stage). More generally, "changes in PRN state" describe joint adjustments in molecule concentrations in anticipation of or response to changing conditions, including dysregulation (i.e., allostatic state). Variation in PRN state occurs within individuals over time, whereas variation in PRN structure occurs across species and populations (see below). 
PRN structure - The species-specific configuration of PRN molecules, their regulatory relationships, and the strengths of these relationships, all of which together determine how the PRN functions. Variation in PRN structure occurs across species, whereas variation in PRN state occurs within individuals over time. We include strengths as part of PRN structure because the organization of regulatory relationships changes very slowly over evolutionary time, and variation in the strengths is a critical aspect of how system function evolves.

Regulatory algorithm - The complete set of biochemical determinants regulating the concentration of a single PRN molecule (i.e., how a PRN molecule is regulated as a function of all its upstream determinants). Conceptualizing this as an algorithm is useful for understanding how it might evolve and affect PRN structure. Thus, regulatory algorithms summarize the information in molecule-focused subsets of PRN structure.

Subnetwork - A part of a PRN that is both highly connected and responsible for a particular aspect of physiological function (e.g., the immune system, energy metabolism).

Symmorphosis - The property of multiple morphological components of a system jointly scaled to their maximal functional capacity, thereby resulting in an economy of design without excess and unusable capacity in any one component

System-level property - A property of a PRN that is determined by its structure and thus cannot be deduced from individual molecules; modularity, link density, and robustness are examples.

\section{Why study physiological regulation in ecology and evolution?}

In recent years, physiological ecologists have sought to use markers of physiological state (immune molecules, hormones, oxidative stress indicators, and so forth) both as proxies for 
organismal condition and to understand how ecological and evolutionary processes may be mediated by individual physiology [1-4]. While sometimes fruitful, the results of many such studies have proven confusing, appearing to depend very precisely on the details of experimental condition $[5,6]$. We believe that these contradictory results are a consequence of treating the physiological markers as independent indicators of some aspect of functioning, rather than as regulatory components of a single unified system responsible for maintaining organismal homeostasis, what we call a physiological regulatory network (PRN). Here, we propose that the systems biology approach implied by PRNs has broad implications for our understanding both of how physiology may constrain or facilitate organismal evolution and of how organisms respond to multiple changes in their environment.

For example, we can consider the biochemical mechanisms responsible for maintaining redox homeostasis and limiting oxidative damage as a single physiological system. However, the role of this system in aging and the evolution of lifespan [7] depends on its interaction with many systems, including intracellular regulation of proton gradients [8], hormonal regulation [9], dietary intake of micronutrients [10], and the use of certain parts of the immune system [11]. Additionally, low levels of oxidative damage are easily tolerated (or even necessary) for most organisms [12]. Health consequences may occur only when control mechanisms are sufficiently disrupted, and disruption tolerance appears to vary substantially across species [13]. Thus, maintenance of oxidative homeostasis depends on many factors not directly implicated in the biochemical cascades resulting in or buffering the effects of oxidation, and all of these factors must be considered together in order to understand how oxidative damage might affect the evolution of lifespan. 
The study of organismal physiology can benefit greatly from an explicit network perspective, much as other biological systems such as gene regulation $[14,15]$ and species interactions $[16$, 17] have benefited from network analysis and modeling. Here we propose that PRNs are critical not just for understanding organismal biology, but also for understanding ecological and evolutionary processes, largely due to the extensive regulatory connections among PRN molecules and the resulting non-independence of ecological influences and selection and on specific traits at ecological and evolutionary timescales, respectively. For example, the vertebrate immune system has evolved alternatives to dealing with different types of parasites (i.e., intra- and extra-cellular, micro and macro) [18], and the relative capacities for these responses depend on genes, diet, internal physiological state, and external environmental conditions. Accordingly, some knowledge of the relevant PRNs is necessary to understand how diet, pathogen environment, and evolutionary history interact to influence host-parasite coevolution.

Much as the fields of genetics and evo-devo have, via recognition of genetic and developmental constraints, reshaped our understanding of the relationship between selection and phenotypic evolution, we believe that a network-based approach to physiology will be critical in identifying physiological constraints on organismal evolution. The importance of PRNs for understanding organismal homeostasis and evolution is analogous to the importance of ecological networks for understanding a variety of community properties including organization, persistence, assembly, robustness, and function. In both cases, the study of complex networks of interacting components can provide novel insights into system-level stability and function not revealed by pairwise or small-motif studies [19]. There is excellent evidence both for the 
regulatory complexity that implies the existence of PRNs and for the importance of this complexity in determining ecological and evolutionary processes [20]. However, only small portions of PRNs have been successfully mapped, and we are not yet in a position to demonstrate the results of PRN studies. Accordingly, most examples we give are at least partly hypothetical. Our goal is to stimulate thought, debate, and novel research directions. Because some of the concepts and terms we use will be new for readers, we recommend reading the glossary before continuing.

\section{What are Physiological Regulatory Networks (PRNs)?}

Networks are central to many aspects of biology. Gene regulatory networks are crucial for adjustment of protein production levels. Protein networks are crucial for maintenance of intracellular homeostasis. Ecological networks mediate species persistence and co-existence as well as ecosystem function. In each of these cases, there are important system-level properties that can be understood only by examining the structure and dynamics of interactions among network components.

In PRNs, homeostasis and performance are determined by interactions among molecules. Each organism has numerous physiological systems composed of interconnected networks of molecules: examples include cytokine cascades in the immune system, the stress responses, lipid balance, energy metabolism, and oxidative balance. These molecular-level networks result in organism-level function (much as species-level networks give rise to ecosystem-level function) and are "homeostatic" in that they allow the phenotype to remain viable in response to a changing internal or external environment. They are not independent of each other, but 
rather communicate through various regulatory pathways, and thus can be considered subnetworks of a larger network, a PRN (Fig. 1). Many of the linkages between these subnetworks are well documented [20]. The non-independence of the subnetworks provides the rationale for integrating them into a comprehensive PRN. We exclude intra-cellular and intra-tissue networks from our definition except insofar as they have important regulatory relationships with the organism-level PRN.

PRNs achieve multiple coherent phenotypic outcomes such as sufficient immune responses, appropriate energy metabolism, and season- and situation-specific behavioural patterns. Many PRN molecules simultaneously affect multiple such outcomes. These "integrators," principally hormones such the sex steroids and glucocorticoids, but also some cytokines, are key regulatory molecules that adjust PRN state according to internal and external conditions [20]. Integrators interact with multiple systems and each other to ensure that phenotypic form and function match environmental conditions and evolutionary priorities (e.g., breeding, clearance of a parasite, fattening to promote migration; Fig. 1). Different aspects of PRNs and integrator regulation are involved in response to predictable changes (such as growth and seasons) and unpredictable changes (such as stress). Testosterone, for example, regulates immune functions, influences behavioural interactions, determines the rate and type of sexual tissue maturation, and affects seasonal changes in reproductive physiology and behaviour [1].

The complexity and integration of PRNs suggest that they will have system-level properties not predictable from analysis of any particular constituent molecule or subnetwork (Box 1, Fig. 1). From an evolutionary perspective, we are interested in how these system properties affect traits such as aging rate and evolvability. Additionally, the evolution of PRNs involves 
interactions among component parts, so the entire system must remain functional as any changes occur. The concept of symmorphosis proposes that PRN nodes will have evolved in concert, with surplus capacity in any component reduced over time due to high maintenance costs. For example, maximal whole-animal oxygen consumption matches rate of oxidative phosphorylation in the mitochondria, delivery rate of oxygen to cells in the microcirculation, and transport rate of oxygen in the blood across a broad range of mammals [21]. Thus, evolution of energy metabolism can occur via changes in capacity at multiple interacting steps in the oxygen transport pathways (see also Box 2). Studying such systems with the PRN concept in mind will facilitate identification of PRN molecules that are pressure points for selection.

\section{Ecological and evolutionary implications of PRNs}

Physiological constraints, correlated traits, and evolvability The complexity and integration of PRNs, including the high degree of correlation among traits, provide for selective responses to changing conditions that can cascade throughout the system [22]. For example, faced with a drop in the abundance of a preferred resource, a species might shift to an alternative resource that happens to have a different vitamin E content. Vitamin E has multiple physiological roles, including as an antioxidant and regulator of the immune system [23]. Hypothetically, a shift in vitamin E intake might affect regulation of oxidative balance as well as response to a parasite. Changes in regulatory roles of vitamin E might ensue; however, such changes might in turn have ripple effects on other PRN-mediated actions such as inflammation and lipid oxidation [24]. It is thus possible that a simple diet shift would require major modifications throughout the PRN. A network approach is useful for tracing both indirect and direct effects throughout systems, as 
seen in ecological network analyses $[25,26]$. In addition, ecological network models have shown the importance of adaptive behavior at the component level for organization, stability, and function at the system level [27]. Such models have been used to identify key constraints on the assembly and evolution of persistent complex networks [28-31]. Similar modeling approaches may prove useful in a PRN context.

Although constraints likely affect PRN evolution, PRN structure might also facilitate evolvability [32]. Canalization and compartmentalization in development have allowed the evolution of diverse structures in ways that correspond to selective pressure on morphology [33, 34]. For example, despite the complexity of networks of gene expression involved in limb development in vertebrates, a small number of T-box genes appear to control differences in limb development, facilitating morphological change in response to selection [35, 36]. Similar principles could apply to the evolution of PRN structure. For instance, modularity (Box 1) might facilitate evolvability by favoring adaptive phenotypic change in simple, coordinated ways. On the other hand, alternative physiological structures (Box 2) might cause divergent PRN evolutionary trajectories even under similar selective conditions, complicating the evolution of highly evolvable physiological modules. It is not known what trade-offs might exist between evolvability and production of an integrated, functioning phenotype, nor how both might be constrained by chance and regulatory complexity.

Regulatory algorithms and local optima An important functional task of a PRN is to help the organism respond appropriately to changing internal or external conditions. This need sets up a potential trade-off between incorporating as much information as possible into a response to environmental change versus the functionality (and evolvability) of the PRN. This trade-off can 
be conceptualized and modeled with "regulatory algorithms." Direct regulatory relationships between PRN molecules are, by definition, determined by the biochemical properties of the molecules involved. The concentration of each molecule is a combined function of all of its upstream regulatory relationships, relationships that together form an "algorithm" that determines the concentration of the molecule, keeping it as close to optimal as possible given the information contained in other molecules about internal and external conditions. The regulatory algorithm concept is similar to that of a "sink web" in ecological network research, which is a subset of a full food web that traces all chains of resource inputs to a particular consumer species.

Natural selection will adjust regulatory algorithms, although there are likely constraints on establishing new biochemical pathways and affinities, which change only very slowly [37]. Modeling the evolution of regulatory algorithms is a promising approach to understanding the balance of selection and constraints, as well as trade-offs between maximizing information content (optimal short-term regulation) and longer-term system evolvability. While simple algorithms (i.e., local optima) appear to be the rule, there are also clear examples of regulatory complexity in physiology: (1) There are immune receptors for polyphenols [38] (implying incorporation of minor and environmentally unpredictable molecules in immune regulation), and (2) Th2:Th1 regulation in chickens incorporates not just carotenoids, vitamin E, and fatty acids, but interactions among them [39, 40]. Two underexplored questions with large implications for PRN evolution are thus how general these complex, specific algorithms are and what conditions produce them. 
As an example of the trade-offs involved in regulatory algorithms, many birds use both photoperiod and temperature to cue reproduction, with luteinizing hormone concentrations controlled via the CNS to trigger timing [41], resulting in an algorithm such as "when photoperiod exceeds threshold $\mathrm{X}$ and temperature exceeds threshold $\mathrm{Y}$, start breeding." However, availability of key resources fed to nestlings could provide additional information to optimize timing (e.g. through diet micronutrient content); to our knowledge, this information is not incorporated into reproductive timing algorithms of most birds, with the likely result of substantial lost reproduction in years with aberrant food supplies. This is probably because the resulting algorithm would be too complex to maintain well given the inter-population variation in food resources. Photoperiod, temperature at start of reproduction, and resource availability all vary across populations within species, so the algorithm needs to be (and is [41]) adjusted at short evolutionary timescales. Thus, a globally optimal algorithm might be something like, "If photoperiod less than $A$ do not start reproducing. If photoperiod greater than $A$ but less than $B$, then if the exponentially weighted sum of temperature and food cue exceeds X start reproducing. If photoperiod greater than B, start reproducing." However, because most of the relevant timing information is contained in photoperiod and temperature, the force of selection on incorporating food availability cues (and their interactions with the other cues) is only as strong as their marginal contribution to the algorithm, likely too weak to adjust well across populations and their changing environments.

PRNs, aging, and evolution Biologists still have no clear understanding of the mechanisms of aging, and existing evolutionary theories do not account for the distribution of aging mechanisms and lifespan across phyla, classes, and orders [42]. If dysregulation of PRNs causes 
aging, the evolution of PRN structure would be tightly linked to the evolution of aging rate. Aging rate (and scope for selection to act on aging rate) should be affected by system-level properties of PRNs (Box 1). For example, species with a highly robust PRN structure would age slowly; since large-scale remodeling of PRN structure might not be feasible at short evolutionary timescales, PRN structure might impose a substantial constraint on aging evolution. Ancestral mammals likely evolved a PRN structure suited to short-lived, high-metabolism animals; this structure might continue to limit human lifespan even while traditionally longer-lived taxa such as turtles and sturgeon evolved long lifespans under appropriate selective regimes [42].

PRN state changes and changing environment as a constraint PRNs do not remain in a fixed state throughout an organism's lifespan; in fact, a key function of PRNs is to facilitate transitions among phenotype states via appropriate adjustments of integrators [20]. At short timescales these shifts might occur between states such as hungry or satiated or stressed and non-stressed. At intermediate scales, there are shifts between seasons or between breeding and nonbreeding. At longer scales, there are developmental shifts between juvenile and adult, or even between sexes in some fish or between worker and queen in some hymenoptera. Much as Piersma and van Gils suggest more generally [43], we hypothesize that the need for increased flexibility between major PRN states (e.g. winter vs. summer, drought vs. normal, stressed vs. normal) is an evolutionary constraint, and that flexibility might trade off with robustness such that an organism living in a relatively constant environment will evolve a more robust PRN structure and thus a longer lifespan. Many system-level properties would seem to be subject to this sort of trade-off, suggesting that the structure of PRNs is a potential mechanism for a tradeoff between flexibility and longevity. This idea might explain the shorter lifespans and faster 
pace of life observed in many temperate bird species compared to tropical residents [44]:

temperate species need to make major physiological shifts either for migration or to survive the winter.

\section{How to study PRNs}

Though the task of studying PRNs may seem daunting, especially since no major portion of a PRN for any species has yet been mapped, the progress made in ecological network research gives reason for optimism. Starting over 100 years ago with the first published image of a food web with 15 highly aggregated taxa [45], there are now have hundreds of ecological networks (of variable resolution and comprehensiveness) with hundreds of species and thousands of interactions represented in a few recent datasets $[46,47]$. At different stages of research, particular insights were gained about food web structure and dynamics that inspired further iterations of improved data collection, new analysis and modeling approaches, and new theory [48]. Similarly, we expect that studies of PRNs will start with incomplete and less-resolved mapping of sub-networks or a few interactions among sub-networks, chosen by researchers based on physiological systems of interest. These studies will yield some basic insights, but will also spark a further round of improved data collection. Given our current abilities to perform large-scale data collection, the potential for integrators to simplify the study of PRNs, and examples and ideas from other areas of network-based research, we expect that PRN research will proceed relatively quickly.

Viewing physiology through a combined network and systems biology lens, as we propose here, will also have concrete effects on the sorts of questions researchers will pose and the 
methods they will need to use. First, alterations will be required in experimental design, analysis, and simulation (Box 3). Second, instead of relying on one or a few biomarkers, it will often be necessary to measure molecules from many PRN subnetworks simultaneously. This approach will necessitate "-omics" methodologies and/or better sample collection techniques allowing measurement of many parameters from small blood samples. Nuclear magnetic resonance [49], PRN-tailored microarrays, dried blood spots [50], and traditional assays are all potential methods, especially in combination.

Third, we will need to pay increasing attention to standardizing methodologies across labs and researchers. For a single molecule, use of the same assay is the main concern, but with PRNs it will also be important to choose the same sets of molecules to measure, a more serious consideration for combining data. At the same time, the complexity of systems-level questions will increasingly require integration of work across research groups, especially to answer comparative and evolutionary questions. Researchers will thus increasingly need to collaborate, and funding organizations may wish to help coordinate efforts.

Fourth, the PRN framework suggests specific research approaches that will be particularly informative for ecological and evolutionary questions. We might identify key molecules within PRN structure (integrators or otherwise) that facilitate or constrain evolutionary change. Such approaches are being used in pharmacology to identify key targets for drug discovery [51] and their potential side-effects [52]. An ecological example would be to ask what bird lineages were able to successfully diversify from tropical habitat into temperate. Are there, perhaps, key differences in PRN structure between lineages that diversified and those that did not that explain the capacity of the system to evolve seasonal flexibility? Another complementary 
approach for studying PRNs is to compare naturally occurring variation in many PRN molecule concentrations across individuals, populations, or species. Repeated evolution of similar PRN structures or particular patterns of variation in PRN molecules should provide clues to how selection acts on PRNs.

\section{Conclusions}

The idea that many physiological systems interact with each other has important implications not typically considered by ecologists and evolutionary biologists. The extension of biologically-focused network research to physiological regulation provides a potentially powerful way to bridge research from gene regulation to ecosystem assembly and function, in terms of organismal evolutionary dynamics. The linkage of many molecules in PRNs could substantially constrain evolutionary change due to the interdependence of molecules and their combined impacts on condition, health, performance, or fitness. However, PRN structure might have evolved specifically to facilitate evolvability; studying the system-level properties of PRNs (Box 1) and integrator networks [20] will help us answer these and related questions. Whatever the answers, PRN thinking will be critical for understanding how organisms respond to environmental cues and how physiological responses feed-up into ecological community structure, dynamics, stability, and evolution.

\section{Acknowledgements}

AAC is member of the FRSQ-funded Centre de recherche clinique Étienne-Le Bel and the Centre de recherche sur le vieillissement, and is supported by NSERC Discovery Grant 402079-2011. 
LBM and SRM are supported by NSF grants 0920475 and 0947177 and 0748349 , respectively. JCW acknowledges grant number IOS-0750540, and the Endowed Chair in Physiology from the University of California, Davis. JAD is supported by NSF grant DBI-0850373.

\section{Box 1: Network analysis and PRN structure}

The characterization of the complex network of regulatory relationships among PRN molecules will provide the empirical basis for evaluating system-level structural properties using methods drawn from network research [53-56]. The simplest characterization of a network includes a set of nodes ("vertices" in graph theory) and the interactions, or links ("edges" in graph theory), between them. Links can be directed ("A affects B") or undirected ("A and B interact"). In PRNs, the links will normally be directed ("A regulates B"). In addition, nodes and/or links can be unweighted ( $A$ is present; a link from $A$ to $B$ is present) or weighted ( $A$ has a certain concentration; the link from A to B has a particular strength). An unweighted, directed network with $n$ nodes can be represented by an $n \times n$ matrix of 0 s and $1 \mathrm{~s}$, where 1 indicates the presence of a link and 0 indicates the absence of a link [57]. The statnet and igraph packages

for $\mathrm{R}$ and the NetworkX library for Python are common software tools for network analysis.

For PRNs, key system-level properties that might be measured using approaches from network structure research include hierarchical structure [58], modularity [59], and connectivity (Fig. 2). A more hierarchical PRN would have greater top-down control but fewer connections among lower-level nodes and less feedback up the chain (Fig. 2b). A more modular PRN would 
have more distinct sub-networks with less feedback among them (Fig. 2c). A more connected PRN would have higher link density, the average links per node $(L / N)$ and/or higher connectance, the proportion of possible links that are realized $\left(L / N^{2}\right)(F i g .2 d)$. Such connectivity measures often serve as simple proxies for network complexity and may relate to other system properties such as robustness to perturbation [60].

These and other system-level properties could reflect whether and how PRNs mediate tradeoffs and evolutionary processes. Evolvability should depend on simplified genetic mechanisms for evolutionary adjustments of PRN structure, which should be facilitated by greater hierarchality and modularity but less connectivity. On the other hand, these traits should also be associated with less information exchange within PRNs, and thus with poorly optimized physiological responses.

\section{Box 2: Alternative physiological regulatory structures}

PRN structure can evolve in different ways to achieve similar physiological outcomes. A simple example is the differing roles of dietary carotenoids on health. Some bird species, such as the Florida Scrub-jay (Aphelocoma coerulescens) and the Waved Albatross (Phoebastria irrorata), have low concentrations of carotenoids, often below the detection limit of standard assays [61]. Other species have much higher concentrations, and for many of these species, including Zebra Finches (Taeniopygia guttata) and Blue and Great Tits (Parus caeruleus and major), there is evidence that individuals with higher concentrations are healthier and/or have greater fitness 
[62]. Thus, it appears that the roles for which carotenoids are critical in one species (e.g. antioxidant defence, immune regulation) are fulfilled by other means in other species.

Another well-studied example is evolutionary adjustment of receptor and enzyme concentrations in testosterone signalling pathways. Actions of testosterone on the brain can depend upon gonadal sources of the hormone in the breeding season and then on central (brain) sources in the non-breeding season. These adjustments can be further regulated by changing sensitivity to the hormone by changing receptors and/or enzymes that convert the hormone to an inactive form or synthesize it de novo in specific cells [1].

Thus, alternative physiological structures have evolved at both short and intermediate evolutionary timescales; however, not all aspects of physiology have different potential structures. In birds, fatty acid composition during migration appears to be adjusted to optimize energy-to-weight ratio, even at a cost of increased oxidative damage [63]. The strong force of selection on weight during migration will thus likely result in conserved or convergent evolution of fatty acid composition, rather than in alternative strategies for fatty acid metabolism, though the details have yet to be confirmed.

While alternative physiological structures are equivalent for short-term function and fitness, they might affect long-term trajectories of physiological evolution because they might open up or close off different possibilities for subsequent changes to PRN structure. For example, a novel physiological role for carotenoids would be much more likely to evolve in Zebra Finches than in Florida Scrub-jays.

\section{Box 3: Analytical methods for PRNs}


The systems biology perspective suggested by PRNs implies that large numbers of physiological parameters will need to be measured and analyzed in concert. Traditional statistical tools such as regression models are incapable of dealing with high dimensionality, complex interactions, and system dynamics. While there is no silver-bullet analytical method currently available [64], a combination of different approaches is slowly yielding results in cellular systems biology.

The first challenge is to characterize PRN structure. Basic biochemical knowledge is an important place to start, and can be augmented with various statistical approaches. Data reduction methods (e.g., principal components analysis) can be used to reduce dimensionality where appropriate. Path analysis and structural equation models can be used to disentangle physiological pathways from observational data, especially if the data are longitudinally structured [65]. Vector field editing uses matrix algebra to track phenotype trajectories through multivariate physiological states, and response surface regression may help identify the affects of multiple integrators on other integrators [20]. Multivariate distribution approaches such as Mahalanobis distance [66] may also help identify individuals with abnormal PRN states.

Once a PRN structure has been identified - even partially - network analysis can be used to identify system-level properties [53, 56]. Additionally, structural equation models and path analysis can be applied to a mapped network to measure the relative importance of direct pathways ("A regulates $B$ ") and indirect pathways ("A to $C$, when $A$ regulates $B$ regulates $C$ "). In ecological systems, indirect and diffuse effects can be as or more important than direct effects $[25,67]$. 


\section{Literature Cited}

1 Hau, M. and Wingfield, J.C. (2011) Hormonally-regulated trade-offs: evolutionary variability and phenotypic plasticity in testosterone signaling pathways. In Molecular mechanisms of life history evolution (Flatt, T. and Heyland, A., eds), pp. 349-362, Oxford University Press

2 Wikelski, M. and Ricklefs, R.E. (2001) The physiology of life histories. Trends in Ecology \& Evolution 16, 479-481

3 Adamo, S.A. (2004) How should behavioural ecologists interpret measurements of immunity? Animal Behaviour 68, 1443-1449

4 McGraw, K.J., et al. (2010) The ecological significance of antioxidants and oxidative stress: a marriage between mechanistic and functional perspectives. Functional Ecology 24, 947-949 5 Matson, K.D., et al. (2006) No simple answers for ecological immunology: relationships among immune indices at the individual level break down at the species level in waterfowl. Proceedings of the Royal Society B: Biological Sciences 273, 815-822

6 Costantini, D. and Verhulst, S. (2009) Does high antioxidant capacity indicate low oxidative stress? Functional Ecology 23, 506-509

7 Beckman, K.B. and Ames, B.N. (1998) The free radical theory of aging matures. Physiological Reviews 78, 547-581

8 Brand, M.D., et al. (2004) Mitochondrial superoxide: production, biological effects, and activation of uncoupling proteins. Free Radical Biology and Medicine 37, 755-767

9 Lin, H., et al. (2004) Oxidative stress induced by corticosterone administration in broiler chickens (Gallus gallus domesticus) 1. Chronic exposure. Comparative Biochemistry \& Physiology B 139, 737744

10 Bertrand, S., et al. (2006) Carotenoids modulate the trade-off between egg production and resistance to oxidative stress in zebra finches. Oecologia 147, 576-584

11 Costantini, D. and Dell'Omo, G. (2006) Effects of T-cell-mediated immune response on avian oxidative stress. Comparative Biochemistry \& Physiology Part A 145, 137-142

12 de Magalhães, J.P. and Church, G.M. (2006) Cells discover fire: Employing reactive oxygen species in development and consequences for aging. Experimental Gerontology 41, 1-10

13 Andziak, B., et al. (2006) High oxidative damage levels in the longest-living rodent, the naked mole-rat. Aging Cell 5, 463-471

14 Hasty, J., et al. (2001) Computational studies of gene regulatory networks: In numbero molecular biology. Nature Reviews Genetics 2, 268-279

15 Schlitt, T. and Brazma, A. (2007) Current approaches to gene regulatory network modelling. BMC Bioinformatics 8, S9

16 Bascompte, J. (2009) Disentangling the web of life. Science 325, 416-419

17 Dunne, J.A. (2009) Food webs. In Encyclopedia of Complexity and Systems Science (Meyers, R.A., ed), pp. 3661-3682, Springer

18 Pulendran, B. (2004) Modulating Th1/Th2 responses with microbes, dendritic cells, and pathogen recognition receptors. Immunologic Research 29, 187-196

19 Stouffer, D.B. and Bascomtpe, J. (2010) Understanding food-web persistence from local to global scales. Ecology Letters 13, 1540161

20 Martin, L.B., et al. (2011) Integrator networks: illuminating the black box linking genotype and phenotype. Integrative and Comparative Biology 51, 514-527

21 Weibel, E.R., et al. (1991) The concept of symmorphosis: a testable hypothesis of structurefunction relationship. Proceedings of the National Academy of Sciences 88, 10357-10361

22 Lande, R. and Arnold, S.J. (1983) The measurement of selection on correlated characters.

Evolution 37, 1210-1226 
23 Han, S.N., et al. (2000) Vitamin E supplementation increases T helper 1 cytokine production in old mice infected with influenza virus. Immunology 100, 487-493

24 Berliner, J.A., et al. (1995) Atherosclerosis: Basic Mechanisms : Oxidation, Inflammation, and Genetics. Circulation 91, 2488-2496

25 Borrett, S.R., et al. (2010) Rapid development of indirect effects in ecological networks. Oikos 119, 1136-1148

26 Williams, R.J., et al. (2002) Two degrees of separation in complex food webs. Proceedings of the National Academy of Sciences 99, 12913-12916

27 Valdovinos, F.S., et al. (2010) Consequences of adaptive behaviour for the structure and dynamics of food webs. Ecology Letters 13, 1546-1559

28 Guill, C. and Drossel, B. (2008) Emergence of complexity in evolving niche-model food webs. Journal of Theoretical Biology 251, 108-120

29 Law, R. and Morton, R.D. (1996) Permanence and the Assembly of Ecological Communities.

Ecology 77, 762-775

30 Loeuille, N. and Loreau, M. (2005) Evolutionary emergence of size-structured food webs.

Proceedings of the National Academy of Sciences of the United States of America 102, 5761-5766

31 Stegen, J.C., et al. (2012) Evolving ecological networks and the emergence of biodiversity patterns across temperature gradients. Proceedings of the Royal Society B: Biological Sciences 279, 1051-1060

32 Kirschner, M. and Gerhart, J. (1998) Evolvability. Proceedings of the National Academy of Sciences of the United States of America 95, 8420-8427

33 Garcia-Bellido, A., et al. (1976) Developmental compartmentalization in the dorsal mesothoracic disc of Drosophila. Developmental Biology 48, 132-147

34 Waddington, C.H. (1942) Canalization of Development and the Inheritance of Acquired

Characters. Nature 150, 563-565

35 Gibson-Brown, J.J., et al. (1996) Evidence of a role for T-box genes in the evolution of limb morphogenesis and the specification of forelimb/hindlimb identity. Mechanisms of Development 56, 93-101

36 Losos, J.B. (1990) The Evolution of Form and Function: Morphology and Locomotor Performance in West Indian Anolis Lizards. Evolution 44, 1189-1203

37 Rosenquist, M., et al. (2000) Evolution of the 14-3-3 Protein Family: Does the Large Number of Isoforms in Multicellular Organisms Reflect Functional Specificity? Journal of Molecular Evolution 51, 446-458

38 Youn, H.S., et al. (2006) Suppression of MyD88- and TRIF-dependent signaling pathways of tolllike receptor by (-)-epigallocatechin-3-gallate, a polyphenol component of green tea. Biochemical Pharmacology 72, 850-859

39 Selvaraj, R.K., et al. (2006) Dietary lutein and fat interact to modify macrophage properties in chicks hatched from carotenoid deplete or replete eggs. Journal of Animal Physiology and Animal Nutrition 90, 70-80 40 Klasing, K.C. (2011) Environmentally induced stochasticity of adaptive immunity. Good, bad, or ugly? In Annual Meeting of the Society for Integrative and Comparative Biology 41 Silverin, B., et al. (2008) Ambient temperature effects on photo induced gonadal cycles and hormonal secretion patterns in Great Tits from three different breeding latitudes. Hormones and Behavior 54, 60-68

42 Finch, C.E. (1990) Longevity, Senescence, and the Genome. University of Chicago Press

43 Piersma, T. and van Gils, J.A. (2011) The Flexible Phenotype: a Body-centred Integration of Ecology, Physiology, and Behaviour. Oxford University Press

44 Wiersma, P., et al. (2007) Tropical birds have a slow pace of life. Proceedings of the National Academy of Sciences 104, 9340-9345 
45 Camerano, L. (1880) On the equilibrinm of living beings by means of reciprocal destruction. In Reprinted in: Lecture Notes in Biomathematics (Levin, S., ed), pp. 360-380

46 Jacob, U., et al. (2011) The role of body size in complex food webs: A cold case. Advances in Ecological Research 45, 181-223

47 Pocock, M.J.0., et al. (2012) The Robustness and Restoration of a Network of Ecological Networks. Science 335, 973-977

48 Dunne, J.A. (2006) The Network Structure of Food Webs. In Ecological networks: linking structure to dynamics in food webs (Pascual, M. and Dunne, J.A., eds), pp. 27-92, Oxford University Press 49 Wang, Y., et al. (2008) Global metabolic responses of mice to Trypanosoma brucei brucei infection. Proceedings of the National Academy of Sciences 105, 6127-6132

$50 \mathrm{McDade}, \mathrm{T}$., et al. (2007) What a drop can do: Dried blood spots as a minimally invasive method for integrating biomarkers into population-based research. Demography 44, 899-925

51 Fliri, A.F., et al. (2010) Cause-effect relationships in medicine: a protein network perspective.

Trends in Pharmacological Sciences 31, 547-555

52 Brouwers, L., et al. (2011) Network Neighbors of Drug Targets Contribute to Drug Side-Effect

Similarity. PLoS ONE 6, e22187

53 Cohen, R. and Havlin, S. (2010) Complex Networks: Structure, Robustness and Function.

Cambridge University Press

54 Newman, M.E.J. (2010) Networks: An Introduction. Oxford Unviersity Press

55 Pamplona, R. and Costantini, D. (2011) Molecular and structural antioxidant defenses against oxidative stress in animals. American Journal of Physiology - Regulatory, Integrative and Comparative Physiology 301, R843-R863

56 West, D.B. (2001) Introduction to Graph Theory. Prentice Hall

57 Mason, O. and Verwoerd, M. (2007) Graph theory and networks in Biology. Systems Biology, IET 1, 89-119

58 Clauset, A., et al. (2008) Hierarchical structure and the prediction of missing links in networks. Nature 453, 98-101

59 Newman, M.E.J. (2006) Modularity and community structure in networks. Proceedings of the National Academy of Sciences 103, 8577-8582

60 Dunne, J.A., et al. (2002) Network structure and biodiversity loss in food webs: robustness increases with connectance. Ecology Letters 5, 558-567

61 Cohen, A.A. and McGraw, K.J. (2009) No simple measures for antioxidant status in birds:

Complexity in inter- and intraspecific correlations among circulating antioxidant types. Functional

Ecology 23, 310-320

62 Blount, J.D., et al. (2003) Carotenoid modulation of immune function and sexual attractiveness in zebra finches. Science 300, 125-127

63 McWilliams, S.R., et al. (2004) Flying, fasting, and feeding in birds during migration: a nutritional and physiological ecology perspective. Journal of Avian Biology 35, 377-393

64 Kitano, H. (2002) Systems Biology: A Brief Overview. Science 295, 1662-1664

65 Shipley, B. (2000) Cause and correlation in biology: a user's guide to path analysis, structural equations and causal inference. Cambridge University Press

66 Mahalanobis, P.C. (1936) Mahalanobis distance. Proceedings National Institute of Science of India 49, 234-256

67 Yodzis, P. (2000) Diffuse effects in food webs. Ecology 81, 261-266

Figure 1: A simplified, partial schematic of a physiological regulatory network (PRN). Red

arrows indicate top-down control, such as steroid hormone modulation of immune function. 
Purple arrows indicate feedback effects, such as antioxidant effects on glucocorticoids. Light blue arrows indicate direct interactions among sub-networks, such as immune regulation by dietary antioxidants. Green arrows indicate direct effects of the environment on sub-networks, such as content of antioxidants in the diet. Yellow arrows indicate environmental regulation of integrators, usually via the CNS. System-level properties of the PRN exist at different levels, including state within individuals (e.g. dysregulation) and species-level structure (modularity). Likewise, phenotype can include individual- or species-level traits (e.g. health and evolvability, respectively). Modularity is determined by the proportion of potential light blue arrows present; interconnectedness by the total number of arrows relative to molecules; and robustness by the density of purple arrows resulting in negative feedback effects. Temporal dynamics and metabolite flux (not shown) can also be important determinants of system-level properties such as dysregulation. The particular structure of connections, as well as their strengths and interactions, will determine how the PRN responds at an individual level and evolves at the species level in response to a changing environment [20].

Figure 2: Some system-level properties of PRNs that can be measured with network theory. A simple hypothetical PRN sub-network is shown (a cytokine cascade, for example). Relative to a baseline scenario (a), we can posit PRN structures that are more hierarchical (b), more modular (c), and that have higher connectivity (d). 